\title{
The Construction of Intelligent Transport System Based on Internet of
}

\section{Things}

\author{
Liping Su ${ }^{1}$, Dong Chen ${ }^{2, *}$ \\ ${ }^{1}$ Guangxi Technological College of Machinery and electricity, Nanning, 530007 \\ ${ }^{2}$ Guangxi Teachers Education University, Nanning, 530023 \\ Nanning, P.R. China \\ * Corresponding author : hgccd @163.com
}

\begin{abstract}
Key words: Internet of things Intelligent transport Information system
\end{abstract}
Abstract: The application of the internet of things in the urban transportation is an important development of the intelligent transportation system in the future. Intelligent traffic system based on the Internet of Things is constructed in this paper in the full understanding of dynamic sensing, active management, and human-vehicle-road collaborative concept, with regards to the present situation and existing problems in the intelligent transportation system. A framework for intellige nt transportation system and its functions are described at last.

\section{Introduction}

With the acceleration of urbanization, motorization, the pace of modernization, the urban population increase, growing faster vehicles, urban traffic congestion and clogging growing, urban transport system overwhelmed, the consequent environmental noise, air pollution, energy waste and other factors plaguing today's transportation in major cities, has become a serious problem around the world now industrial countries and developing countries. So, faced with in today's world of globalization, information technology development trend, the traditional means of transportation technology and no longer meet the requirements of economic and social development, intelligent transportation is the inevitable choice for urban transport development, is a revolution in urban transport undertakings. Things appear to intelligent transportation industry breakthrough brings rare opportunities to bring new horizons for the development of intelligent transportation, smart transportation to provide a wider space for development, and therefore modern urban transport calling for "Internet of things " [1]. "The new generation of intelligent transportation" development provides important technical support for the realization of real-time, efficient, accurate, safe, energy-saving intelligent transport objectives and provide technical support for the Internet of Things technology, networking technology will bring a intelligent urban traffic The new upgrade.

\section{Internet of things}

\section{1 definition}

Internet of things to be considered following the world's information industry, the third wave of Internet, mobile communication network after, is an important part of a new generation of information technology. Internet of things refers to information through a variety of sensing devices, such as sensors, radio frequency identification (RFID) technology, global positioning systems, infrared sensors, laser scanners, gas sensors and other devices and technologies, real-time collection of any need to monitor, connection, object or interactive process, collecting the information needs of its sound, light, heat, electricity, mechanics, chemistry, biology, location, combined with a vast network formation and the Internet. Its aim is to achieve things and things, things and people, all the items 
connected to the network, to facilitate the identification, management and control ${ }^{[2]}$. Its main feature is to get the physical world through RFID, sensors, etc. all kinds of information, combined with the Internet, mobile communication network and other network transmission and interactive information, the use of intelligent computing technology information analysis and processing, thereby improving the material world perception, intelligent decision-making and control.

\subsection{Internet of things development situation in the transport sector}

Internet of things in the field of transport is widely used, including applications, vehicle identification, container industry, intelligent vehicle access control system, railway transportation management and vehicle identification and RFID taxi management system application structure.

Currently, in our country, things in the field of urban intelligent transportation applications have been developed, such as the scheduling aspect of the vehicle. Through intelligent transportation infrastructure of the entire city road vehicles and real-time information collected, through the intelligent control center, information on these calculations, to provide the best solutions and route traffic control ${ }^{[3-7]}$.

\section{Things construct based Intelligent Transportation System}

Intelligent transportation system should be based on the traditional intelligent, more integrated into the user-friendly design and management philosophy, using smarter methods, including the application of intelligent recognition, data integration, mobile computing, cloud computing and other information technology for traffic management and traffic participants to provide more scientific decision support and more personalized service. Make full use of IT comprehensive sensing, integration of critical information systems for the transport sector to run core analysis of daily travel, transportation, traffic safety and public service needs to make intelligent response.

Under the guidance of the "dynamic perception, active management, collaborative way of people and vehicles," the development of the concept, advanced networking technology used in intelligent transportation of things based on intelligent transportation systems, through organic and full inclusion of intelligent transportation and Things using the concept of things enrich the connotation of intelligent transportation, which made the new generation of intelligent transportation system framework.

\subsection{System building ideas}

In the planning and design of intelligent transportation systems framework, while organically things into being intelligent transportation system framework. First, the Internet of Things perception layer into the base layer and the data layer, sensor nodes and outfield equipment intelligent transportation intelligent transportation system integration, sensory information and intelligent transportation data integration. The second is the network layer things into the base layer of intelligent transport systems, sensor networks and intelligent transportation peripherals peripheral acquisition system integration, core bearer networks and intelligent transportation and communication systems integration. Third, the perception of the center of things and intelligent traffic data layer and application layer combining both perception Center Intelligent Traffic Management Center data, but also the traffic information service distribution center.

\subsection{Construction of the target system}

By promoting networking applications in the transport sector, in order to achieve the following objectives:

(1) To achieve a more thorough perception of the various elements in the collection of traffic signals.

(2)Transportation of the elements to achieve a more comprehensive interoperability.

(3)To achieve deeper in the operation and management of intelligent transportation. 


\subsection{System Architecture System}

The main floor is divided into information perception, information processing layer, network layer, service layer and application layer support structure five-layer structure of things Intelligent Transportation System. In the traditional networking system architecture, only the perception layer, transport layer and application layer three-layer structure. Due to the high traffic of information technology projects in data collection frequency, the type of signal acquisition range, resulting in a bear standardization of information, transport layer stress analysis, processing and transmission work is too big, it does not run smooth, so this will be a conventional transmission layer to improve the information processing layer and the network layer two-layer structure, so the system in the perception layer to obtain traffic information, after some processing and then transmitted by the network layer, thereby improving data transmission efficiency and safety. In addition, numerous intelligent transportation subsystems, and each system requires a data vary, so before adding a new business application layer support layer, the depth of excavation and analysis of data is completed, the application layer to provide the required accurate, real-time data. Ensure the efficient operation of the entire system, and ultimately the perception of traffic dynamics, active management and road vehicles collaborative purposes. The overall structure of the system shown in Fig.1:

Perception layer: The main function is to collect information on the perception and, through the RFID tag reader, barcode read-only, sensors, cameras, video equipment, complete networking applications for data acquisition and a system of useful information;

Information processing layer: to complete the analysis and screening information, including clear information garbage, blocking integration and other functions;

Network layer: mainly responsible for the transmission of information to complete the communication between the layers of things, the network basically consists of the Internet, mobile communications, local area networks;

Business Support layers: an integrated data center, cloud computing and other advanced technologies for information integration, classification, and according to different needs and application module application layer of an information transfer point;

Application Layer: Implementing the reverse control command according to the state of the elements of the traffic, the main achievement of traffic monitoring, scheduling, induction, management and a series of functions.
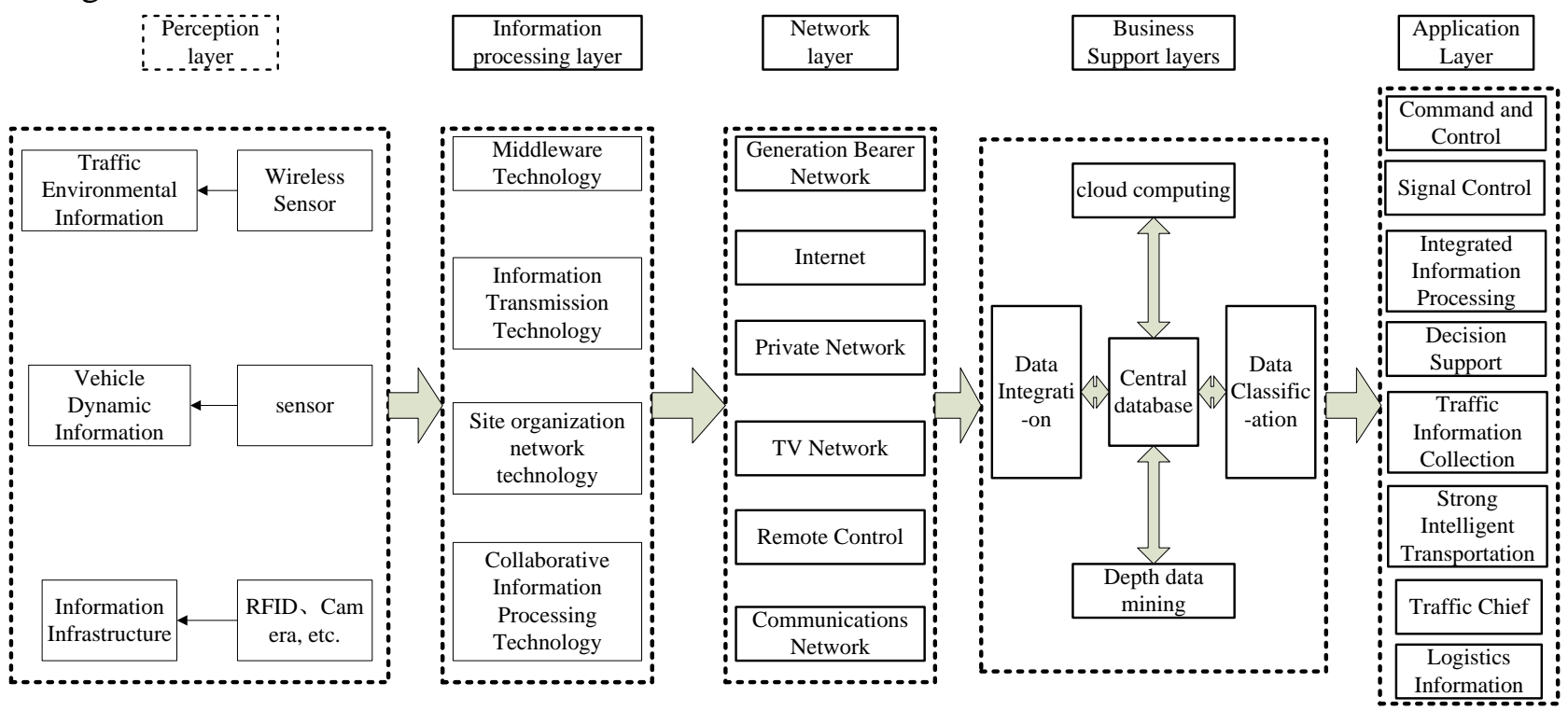

Fig.1 Things based system architecture of Intelligent Transportation Systems 


\subsection{Application System}

With respect to the intelligent transportation system for data collection, transmission, use and feedback, the emphasis on the improvement of Things intelligent transportation system is a real-time information collection, processing and transport to provide accurate and timely decisions. In the operation of intelligent transportation systems, networking technology is mainly reflected in several aspects of dynamic real-time information perception, intelligent monitoring, intelligent scheduling, intelligent management, intelligent decision-making.

Dynamic real-time information perception: in traffic management, real-time acquisition by dynamic traffic conditions, infrastructure and traffic environment and other data, the use of information processing layer networking information for a preliminary order and screening, will be transferred to business support through network layer information layer data unified management and control. Traffic data calculated by means of real-time calculation of various intelligence generated Last Uploaded central database sharing. Thus, the levels of management through the foreground application can view real-time traffic system reflects up data to the scientific traffic scheduling and management.

Intelligent monitoring: Intelligent Transportation Systems through a variety of monitoring equipment for transportation, infrastructure, transportation and environment status monitoring, and data center data upload all types of safety monitoring equipment, by the results of the data analysis, automatically determines traffic conditions and other class security status, automatically implement contingency plans. More critical is the Internet of Things intelligent transportation systems can change based on the trend of each sensor data security, automatic analys is of its development, to find out the causes and solutions, anti loyal to the first place.

Smart Scheduling: dynamic information collection and monitoring, traffic accidents and other emergencies for the speedy implementation of the plan to develop the best, and provide technical support for the implementation of the plan, and after the event processing is complete but also to evaluate the effect of the implementation, in order to constantly improve emergency Decision Support System, to provide a more scientific method of handling related events for the future.

Smart management: Traffic management involves all aspects of the operation of large overall traffic management, small intersection, parking management. Based on the construction of Things intelligent transportation system will eventually converged transport all management information and resources, intelligent management.

Intelligent Decision: to achieve a real-time information in a dynamic perception, intelligent monitoring, intelligent scheduling, after intelligent management, all the information is basically a traffic have achieved the level of interoperability and sharing of data, and this share is real-time, in order to maximize assurance the accuracy of the data. Intelligent transportation system through realtime database, data warehouse and a series of data storage and analysis tools, the use of advanced data analys is model industry, for the storage of various data collection process, the original system of mutual unrelated data into knowledge, then refining into policy recommendations, ultimately leading the entire enterprise to build a command cockpit.

\section{Conclusions}

Things technology in the field of intelligent traffic control, will enhance the information services and intelligent traffic management and control, and provide a strong science and technology security and technical support for the transformation of the traffic management model. With the Internet of Things technology matures and depth, as well as strong government support and a large investment in the construction of intelligent transportation in major cities, can foresee the future perception of form transportation network will make people, cars, road interaction between the three elements 
relations to a new way to present, China's road traffic management intelligence level will reach unprecedented levels, and ultimately real-time, accurate, efficient, safe, smooth and environmentally friendly transport management objectives.

\section{Acknowledgements}

This work was financially supported by "Guangxi university scientific research 《Key technology of dangerous goods transportation based on Internet of things》(YB2014236) research project”and "Guangxi university scientific research 《Research on Key Technologies of Intelligent Transportation Based on Internet of things》 (KY2015LX674) research project”.

\section{References}

[1] Guo Feng. Application of Things technology in Intelligent Transportation System [J]. Applied Science and Technology, 2014 (9): 289

[2] Cai Mei. Overview of Things [J]. Electronic Product Reliability and Environmental Testing, 2011 (1): 59-60

[3] Li Ye, Physiognomy, Dong Libo. Application of Intelligent Transportation of Things [J]. Mobile Communications, 2010 (15): 30-34

[4] Xiaoliang, Mi odd, Peng Su Mian like. Things investigate the application and development of rail transport in the field of [J]. Railway Signalling \& Communication, 2001 (3): 47-48

[5] Li Li, Zhu fresh, Fang. EPC Middleware System [J]. Computer Engineering and Design, 2006 (18): 56-59

[6] Huanghai Kun, Deng Jiajia. Things gateway technology and application [J]. Telecommunications, 2010 (4): 20-24

[7] Comments: the development of things at home and abroad Status and Problems [EB / OL]. [2010 - 3]. http : / / www . wlwcy . ibicn . com / technology / archive / 201.003/ 03381362899146621 1279. html 